\title{
Correction to: Detection and classification of vehicles from omnidirectional videos using multiple silhouettes
}

\author{
Hakki Can Karaimer ${ }^{1,2} \cdot$ Ipek Baris $^{2} \cdot$ Yalin Bastanlar $^{2}$
}

Published online: 22 January 2018

○) Springer-Verlag London Ltd., part of Springer Nature 2018

\section{Correction to: Pattern Anal Applic (2017) 20:893-905 https://doi.org/10.1007/s10044-017-0593-z}

An acknowledgements section was missing in this paper. It should read as follows:

\section{Acknowledgements}

This work was supported by The Scientific and Technical Research Council of Turkey (TUBITAK) Project No: $113 \mathrm{E} 107$.

The authors apologise for this error.

The original article can be found online at https://doi.org/10.1007/ s10044-017-0593-z.

Yalin Bastanlar

yalinbastanlar@iyte.edu.tr

Hakki Can Karaimer

karaimer@eecs.yorku.ca

Ipek Baris

ipekbrs@gmail.com

1 Present Address: Department of Electrical Engineering and Computer Science, Lassonde School of Engineering, York University, Toronto, Canada

2 Department of Computer Engineering, Izmir Institute of Technology, 35430 Izmir, Turkey 